PROCEEDINGS OF THE

AMERICAN MATHEMATICAL SOCIETY

Volume 132, Number 11, Pages 3167-3175

S 0002-9939(04)07508-2

Article electronically published on May 12, 2004

\title{
QUASI-HOMOMORPHISMS AND STABLE LENGTHS IN MAPPING CLASS GROUPS
}

\author{
D. KOTSCHICK
}

(Communicated by Ronald A. Fintushel)

\begin{abstract}
We give elementary applications of quasi-homomorphisms to growth problems in groups. A particular case concerns the number of torsion elements required to factor a given element in the mapping class group of a surface.
\end{abstract}

\section{Introduction}

Let $G$ be a group, and $S \subset G$ an arbitrary subset. The $S$-length $l_{S}(g)$ of $g \in G$ is the minimal number of factors in a factorisation of $g$ into elements of $S$. For a homomorphism $\varphi: G \rightarrow \mathbb{R}$, set $C(\varphi, S)=\sup _{s \in S}|\varphi(s)|$. Then

$$
n \cdot|\varphi(g)|=\left|\varphi\left(g^{n}\right)\right| \leq l_{S}\left(g^{n}\right) \cdot C(\varphi, S) .
$$

If $\varphi(g) \neq 0$ and $\left.\varphi\right|_{S}$ is bounded, then we obtain a positive lower bound for the $S$-length $l_{S}\left(g^{n}\right)$ of $g^{n}$, which is linear in $n$. Hence we obtain a positive lower bound for the stable $S$-length $\|g\|_{S}=\lim _{n \rightarrow \infty} \frac{l_{S}\left(g^{n}\right)}{n}$ of $g$ :

$$
\|g\|_{S} \geq \frac{|\varphi(g)|}{C(\varphi, S)} .
$$

In many important situations there are no homomorphisms from $G$ to $\mathbb{R}$ to which one can apply this argument, either because there are no homomorphisms at all, or they all vanish on a given $g$. However, quasi-homomorphisms can be used in the same way as homomorphisms to find lower bounds on the length. If $\varphi$ is a homogeneous quasi-homomorphism with defect $D(\varphi)$ (see Section 2 below for the definitions), then instead of (10) we have

$$
n \cdot|\varphi(g)|=\left|\varphi\left(g^{n}\right)\right| \leq l_{S}\left(g^{n}\right) \cdot C(\varphi, S)+\left(l_{S}\left(g^{n}\right)-1\right) \cdot D(\varphi),
$$

Received by the editors July 28, 2003.

2000 Mathematics Subject Classification. Primary 20F69; Secondary 20F12, 57M07.

The author is a member of the European Differential Geometry Endeavour (EDGE), Research Training Network HPRN-CT-2000-00101, supported by The European Human Potential Programme.

${ }^{1}$ The length is infinite if no such factorisation exists.

${ }^{2}$ For example if $S$ is finite, or, more generally, falls into finitely many conjugacy classes.

(C)2004 American Mathematical Society 
which again leads to a lower bound for $l_{S}\left(g^{n}\right)$ which is positive and linear in $n$ as soon as $\varphi(g) \neq 0$ and $\left.\varphi\right|_{S}$ is bounded. For the stable $S$-length we then have 3

$$
\|g\|_{S} \geq \frac{|\varphi(g)|}{C(\varphi, S)+D(\varphi)} .
$$

This kind of estimate has been discussed extensively in the literature for the case when $S$ is taken to be the set of commutators in $G$, see Bavard [2] and the papers cited there, and [3, 7, 21] for some more recent developments.

In this paper we apply the estimates (31) and (4) in situations when $S$ is not necessarily the set of commutators. However, as the positivity of the stable commutator length is equivalent to the existence of homogeneous quasi-homomorphisms by Bavard's theorem [2], the stable commutator length provides a kind of universal lower bound for the stable $S$-length for all $S$. We will see in particular that if we take $S$ to be the set of elements of finite order in $G$, then the corresponding stable torsion length is bounded below by twice the stable commutator length.

In the final section we apply our results to mapping class groups of surfaces. We will also exhibit elements $g$ of infinite order in mapping class groups for which the commutator length and the torsion length of $g^{n}$ are constant, and therefore the stable commutator and torsion lengths vanish. For these elements the stable $S$-length is positive for any finite $S$ by a result of Mosher [20] and Farb-Lubotzky-Minsky [8]. Thus, quasi-homomorphisms give lower bounds for the stable $S$-length, but they only tell the whole story when $S$ is the set of commutators. We shall see that quasihomomorphisms on mapping class groups also lead to a unified approach to several other of their properties, which were originally established by other means: failure of (weak) bounded generation [8], non-arithmeticity [12] and superrigidity [9, 13].

\section{QUASI-HOMOMORPHISMS}

A map $f: G \rightarrow \mathbb{R}$ is called a quasi-homomorphism if its deviation from being a homomorphism is bounded; in other words, there exists a constant $D(f)$, called the defect of $f$, such that

$$
|f(x y)-f(x)-f(y)| \leq D(f)
$$

for all $x, y \in G$. Every quasi-homomorphism can be homogenized by defining

$$
\varphi(g)=\lim _{n \rightarrow \infty} \frac{f\left(g^{n}\right)}{n} .
$$

Then $\varphi$ is again a quasi-homomorphism, is homogeneous in the sense that $\varphi\left(g^{n}\right)=$ $n \varphi(g)$, and is constant on conjugacy classes. (Compare [2, Proposition 3.3.1.) The inequality (3) is immediate.

2.1. Commutator lengths. Consider the situation when $S=C$ is the set of commutators. In this case we write $c(g)$ for the commutator length $l_{C}(g)$, and $\|g\|_{C}$ for the stable commutator length.

For any quasi-homomorphism $\varphi$ we have

$$
|\varphi([x, y])| \leq\left|\varphi\left(x y x^{-1}\right)+\varphi\left(y^{-1}\right)\right|+D(\varphi) .
$$

\footnotetext{
${ }^{3}$ Note that the stable $S$-length can be defined and is finite as soon as $g$ has some power that is a product of elements in $S$, even if $g$ itself cannot be factored in $S$.
} 
If $\varphi$ is homogeneous, then $\varphi\left(x y x^{-1}\right)=\varphi(y)$ by constancy on conjugacy classes, and $\varphi\left(y^{-1}\right)=-\varphi(y)$ by homogeneity. Thus $\left.\varphi\right|_{C}$ is bounded by $C(\varphi, C)=D(\varphi)$, and (3) becomes

$$
n \cdot|\varphi(g)|=\left|\varphi\left(g^{n}\right)\right| \leq\left(2 c\left(g^{n}\right)-1\right) \cdot D(\varphi) .
$$

In particular,

$$
\|g\|_{C} \geq \frac{|\varphi(g)|}{2 D(\varphi)} .
$$

Bavard's main theorem in 22] states that taking the supremum over the right hand sides for all homogeneous quasi-homomorphisms gives an exact calculation of the stable commutator length. In particular, if $\|g\|_{C}$ is positive, then there exists a homogeneous quasi-homomorphism $\varphi: G \rightarrow \mathbb{R}$ with $\varphi(g) \neq 0$.

2.2. Torsion lengths. Now we take $S=T$, the set of torsion elements of $G$. We denote the torsion length $l_{T}(g)$ by $t(g)$.

Clearly every homogeneous quasi-homomorphism $\varphi$ vanishes on $T$. Therefore we can take $C(\varphi, T)=0$, and we find from (3) that

$$
n \cdot|\varphi(g)|=\left|\varphi\left(g^{n}\right)\right| \leq\left(t\left(g^{n}\right)-1\right) \cdot D(\varphi) .
$$

In particular,

$$
\|g\|_{T} \geq \frac{|\varphi(g)|}{D(\varphi)} .
$$

Comparing (8) and (6), and using the fact that (6) is sharp [2], leads to the following:

Proposition 1. Let $G$ be a group with finitely generated Abelianization. For all $g \in G$ we have $2\|g\|_{C} \leq\|g\|_{T}$.

Proof. Both sides of the inequality are homogeneous, so we can replace $g$ by a power if necessary.

If the stable torsion length of $g$ is defined and finite, then $g$ has a power that is a product of elements of finite order. Therefore every homomorphism $G \rightarrow \mathbb{Z}$ vanishes on $g$. As $G$ is assumed to have finitely generated Abelianization, $g$ must have a power that is in the commutator subgroup. Thus, after replacing $g$ by a suitable power, we may assume that it is a product of commutators and is also a product of torsion elements $t_{1}, \ldots, t_{k}$, with $k=t(g)$ the torsion length.

For arbitrary elements $x, y$ in the commutator subgroup of $G$ one has

$$
\|x y\|_{C} \leq\|x\|_{C}+\|y\|_{C}+\frac{1}{2}
$$

because $(x y)^{n}$ differs from $x^{n} y^{n}$ by at most $\frac{n}{2}+c$ commutators, see [2], Proposition 3.7.1. Applying this repeatedly, we obtain

$$
\|g\|_{C} \leq \sum_{i=1}^{k}\left\|t_{i}\right\|_{C}+\frac{1}{2}(k-1) .
$$

As the stable commutator length of an element of finite order vanishes, we conclude that

$$
t(g)=k \geq 1+2\|g\|_{C} .
$$

Applying this to $g^{n}$ instead of $g$, using the homogeneity of $\|\cdot\|_{C}$, and taking the limit as $n \rightarrow \infty$, we obtain the claim. 
If $g \in G$ is conjugate to some power $g^{n}$ with $n \neq 1$, then clearly every homogeneous quasi-homomorphism vanishes on $g$, and therefore its stable commutator length also vanishes. A special case of such an element is a product of two involutions. In this case we can go further and show the vanishing of the stable torsion length, because every power of $g$ has torsion length at most 2 :

Example 2. Suppose $g=s t$, with $s$ and $t$ of order 2. Then

$$
g^{2 n}=\alpha t \alpha^{-1} \cdot t
$$

with $\alpha=g^{n-1} s=$ stst...sts. As the order of an element is invariant under conjugation, we conclude that $t\left(g^{2 n}\right) \leq 2$. We also have

$$
g^{2 n+1}=\alpha t \alpha^{-1} \cdot t s t=\alpha t \alpha^{-1} \cdot t s t^{-1},
$$

and so $t\left(g^{2 n+1}\right) \leq 2$ as well.

We conclude that for such a product of involutions both the stable torsion length and the stable commutator length vanish.

Example 3. In $S L(2, \mathbb{Z})$ take

$$
g=\left(\begin{array}{ll}
2 & 1 \\
1 & 1
\end{array}\right)=\left(\begin{array}{cc}
0 & 1 \\
-1 & 0
\end{array}\right)\left(\begin{array}{cc}
-1 & -1 \\
2 & 1
\end{array}\right)
$$

Then $g$ has infinite order, but the factors on the right hand side both have order 4. Projecting to $P S L(2, \mathbb{Z})$, these factors become involutions, so by Example2 the image of any power of $g$ in $P S L(2, \mathbb{Z})$ has torsion length at most 2 . It follows that the torsion length of $g^{n} \in S L(2, \mathbb{Z})$ is at most 3 .

In the above example $g$ is conjugate to $g^{-1}$. On the other hand, if an element $g \in S L(2, \mathbb{Z})$ of infinite order is not conjugate to its inverse, then Polterovich and Rudnick 21] proved that there is a homogeneous quasi-homomorphism which does not vanish on $g$. Therefore, our discussion above shows that $g$ has positive stable torsion length.

In Section 3 we will generalize Example 3 to mapping class groups of surfaces of higher genus, by exhibiting elements of infinite order for which the torsion lengths of all their powers are equal to 2 .

2.3. Bounded generation. A group $G$ is said to be boundedly generated if there are finitely many elements $g_{1}, \ldots, g_{k}$ such that any element $g \in G$ can be written in the form $g=\prod_{i=1}^{k} g_{i}^{\alpha_{i}}$ for some $\alpha_{i} \in \mathbb{Z}$. A potentially weaker property is the following:

Definition 4. A group $G$ is weakly boundedly generated by $g_{1}, \ldots, g_{k}$ if for every $g \in G$ there is a number $N$ such that all powers $g^{n}$ can be written in the form

$$
g^{n}=\prod_{i=1}^{N} h_{i}(n)^{\alpha_{i}(n)}
$$

for some $\alpha_{i}(n) \in \mathbb{Z}$, and with each $h_{i}(n)$ conjugate to some $g_{j} \in\left\{g_{1}, \ldots, g_{k}\right\}$.

The point is that the $g_{j}$ may be conjugated, permuted and repeated at will, and that $N$ may depend on $g$, though not on $n$. The independence of $n$ implies that the space of homogeneous quasi-homomorphisms on $G$ is necessarily finite-dimensional:

Proposition 5. If $G$ is weakly boundedly generated by $g_{1}, \ldots, g_{k}$, then the vector space of homogeneous quasi-homomorphisms $\varphi: G \rightarrow \mathbb{R}$ is of dimension at most $k$. 
Proof. Applying $\varphi$ to (9) and using the quasi-homomorphism property $N-1$ times, we find that

$$
\left|\varphi\left(g^{n}\right)-\sum_{i=1}^{N} \varphi\left(h_{i}(n)^{\alpha_{i}(n)}\right)\right| \leq(N-1) D(\varphi) .
$$

Using the homogeneity of $\varphi$ and dividing by $n$, we obtain

$$
\left|\varphi(g)-\sum_{i=1}^{N} \frac{\alpha_{i}(n)}{n} \varphi\left(h_{i}(n)\right)\right| \leq \frac{N-1}{n} D(\varphi) .
$$

As $n$ goes to infinity, the right hand side tends to zero because $N$ is independent of $n$, and so we see that $\varphi(g)$ is determined by the values of $\varphi$ on the $h_{i}(n)$, each of which is, by assumption, conjugate to some $g_{j}$. As $\varphi$ is constant on conjugacy classes, it is completely determined by its values on $g_{1}, \ldots, g_{k}$.

Remark 6 . The conclusion of the proposition can be strengthened if one knows that $\varphi\left(g_{j}\right)$ has to vanish for all $\varphi$, for example because $g_{j}$ is torsion, or is conjugate to one of its powers.

\section{MAPPING CLASS GROUPS}

Consider the mapping class group $\Gamma_{h}$ of isotopy classes of orientation-preserving diffeomorphisms of a compact oriented surface of genus $h$. Maclachlan [17 proved that this is generated by (finitely many) torsion elements. Thus in particular its Abelianization is finite. For $h \geq 3$ it is known that the Abelianization is trivial, i.e., the mapping class group is perfect [22].

By using results about the Seiberg-Witten invariants of symplectic 4-manifolds, it has been proved that the commutator length of a power of a Dehn twist $g \in \Gamma_{h}$ grows linearly with the exponent, see [7] and the elaborations in [4, 14]. The argument admits the following slight generalization:

Theorem 7. Let $\Gamma_{h}$ be the mapping class group of a closed oriented surface $\Sigma_{h}$ of genus $h \geq 2$. If $g \in \Gamma_{h}$ is the product of $k$ right-handed Dehn twists along homotopically essential disjoint curves $a_{1}, \ldots, a_{k} \subset \Sigma_{h}$, then

$$
c\left(g^{n}\right) \geq 1+\frac{n k}{6(3 h-1)} .
$$

Proof. We shall argue as in [4]. As the curves $a_{i}$ are disjointly embedded in $\Sigma_{h}$, we can construct a smooth Lefschetz fibration over the 2-disk $D^{2}$ with precisely one singular fiber $F_{0}$ and with vanishing cycles $a_{1}, \ldots, a_{k}$. The monodromy around the boundary of the disk is $g$. As the Dehn twists along the $a_{i}$ are all right-handed, there is a symplectic (in fact Kähler) structure on the total space compatible with the fibration, cf. [10]. Pulling back the fibration under the map $z \mapsto z^{n}$ and taking the minimal resolution, we obtain a symplectic Lefschetz fibration over $D^{2}$ with only one singular fiber having $k \cdot n$ vanishing cycles, such that there are $n$ parallel copies of each $a_{i}$. The monodromy of this fibration around the boundary of the disk is $g^{n}$. This can be expressed as a product of $c\left(g^{n}\right)$ many commutators, so that we can find a smooth surface bundle with fiber $\Sigma_{h}$ over a surface of genus $c\left(g^{n}\right)$ with one boundary component and the same restriction to the boundary. Let $X$ be the Lefschetz fibration over the closed surface $B$ of genus $c\left(g^{n}\right)$ obtained by gluing together the two fibrations along their common boundary. 
By construction, $X$ is symplectic. As all the $a_{i}$ are homotopically essential, $X$ is relatively minimal, so that we can apply Theorem 8 of [4]. In the notation of [4] we have $D=1$, because there is only one singular fiber, and $N$, the total number of irreducible components of singular fibers, is $N=k \cdot n+c$, with $c$ a constant independent of $n$ which only depends on the configuration of curves $a_{1}, \ldots, a_{k} \subset \Sigma_{h}$. Theorem 8 of [4] gives $k \cdot n \leq 6(3 h-1)\left(c\left(g^{n}\right)-1\right)+6 c$. Pulling back the fibration to large degree coverings of the base, we finally obtain $k \cdot n \leq 6(3 h-1)\left(c\left(g^{n}\right)-1\right)$, as claimed.

Combining Theorem [7] with Proposition [1, we obtain:

Corollary 8. If $g \in \Gamma_{h}$ is the product of $k$ right-handed Dehn twists along homotopically essential disjoint curves, then its stable torsion length is bounded below by

$$
\|g\|_{T} \geq \frac{k}{3(3 h-1)} .
$$

A fortiori, the torsion length is unbounded on $\Gamma_{h}$. This answers a question raised by Brendle and Farb in their recent paper [5]. Motivated by their paper, I had first proved the corollary for powers of a single Dehn twist in a different way. Namely, it is known that $\Gamma_{h}$ contains only finitely many conjugacy classes of elements of finite order 4 . Therefore, the commutator length is bounded on torsion elements. As the commutator lengths of powers of Dehn twists grow linearly with the exponent by the special case of Theorem 7 proved in [7], the torsion lengths must also grow linearly5.

The existence of a non-trivial quasi-homomorphism guaranteed by the special case of Theorem 7 proved in [7] implies that mapping class groups do not have the property $(T T)$ discussed by Monod in Section 13.2 of [19], although it says nothing about Kazhdan's property $(T)$, of which $(T T)$ is a strengthening.

Corollary 8 is a special case of a result which holds for the stable $S$-length for any $S$. Indeed, for products $g$ of commuting right-handed Dehn twists Theorem 7 implies the existence of a homogeneous quasi-homomorphism $\varphi$ with $\varphi(g) \neq 0$. This in turn implies, by the estimate (4), that $g$ has positive stable $S$-length for any $S$ on which $\varphi$ is bounded. If we take for $S$ a (symmetric) finite generating set of the mapping class group, we find that $l_{S}\left(g^{n}\right)$ grows linearly with $n$. This last statement was proved earlier for all elements of infinite order in $\Gamma_{h}$ by FarbLubotzky-Minsky [8], cf. [14. In fact, Farb-Lubotzky-Minsky [8] give a direct argument estimating the word length only for certain reducible mapping classes, and then appeal to earlier work of Mosher [20] for the case when $g$ has a power that is pseudo-Anosov, at least on a subsurface of $\Sigma_{h}$.

There is an important difference between the argument of [8] and ours: the former is insensitive to the chirality of Dehn twists, whereas ours is not. Indeed, if we allow both right-handed Dehn twists and their inverses, then the proof of Theorem 7 breaks down. The corresponding fibration is no longer symplectic, and is not a Lefschetz fibration but an achiral Lefschetz fibration. We now show that this difficulty in the proof of Theorem 7 cannot be overcome by other arguments.

\footnotetext{
${ }^{4}$ This follows from a result of Harvey [11. See 16] for an alternative proof.

${ }^{5}$ Compare also [15].
} 
Example 9. Let $a \subset \Sigma_{h}$ be an essential simple closed curve, and $f: \Sigma_{h} \rightarrow \Sigma_{h}$ a diffeomorphism 6 with $a \cap f(a)=\emptyset$. Let $b=f(a)$, and $g=t_{a} t_{b}^{-1}$, where $t_{c}$ denotes the right-handed Dehn twist along $c$. Then

$$
g^{n}=\left(t_{a} t_{b}^{-1}\right)^{n}=t_{a}^{n} t_{b}^{-n}=\left(f^{-1} t_{b} f\right)^{n} t_{b}^{-n}=\left[f^{-1}, t_{b}^{n}\right],
$$

and thus the commutator length of $g^{n}$ is constant $=1$, although $g$ has infinite order in $\Gamma_{h}$.

If we take for $f$ an involution, then $g$ is the product of the two involutions $f$ and $t_{b} f t_{b}^{-1}$. Thus, by Example2, the torsion length of $g^{n}$ is equal to 2 for all $n \neq 0$. In particular, its stable torsion length vanishes.

The previous example is inspired by the work of McCarthy and Papadopoulos 18. on involutions in mapping class groups. They have also shown that there are products of involutions which are pseudo-Anosov. These are analogous to the Anosov diffeomorphism of the torus in Example 3. They are elements of infinite order in $\Gamma_{h}$ which are conjugate to their inverses, and have trivial stable torsion and commutator lengths, although they have positive stable $S$-length for every finite $S$. This shows that there is no chance to find homogeneous quasi-homomorphisms which are nonzero on an arbitrarily fixed pseudo-Anosov mapping class. Nevertheless, in their proof of the following theorem, Bestvina and Fujiwara [3] have constructed many quasi-homomorphisms which do not vanish on certain pseudo-Anosov elements.

Theorem 10 ([3]). Let $G$ be any non-virtually-Abelian subgroup of a mapping class group. Then the space of homogeneous quasi-homomorphisms on $G$ is infinitedimensional.

Combining this result for the case when $G$ is a mapping class group itself with Proposition 5, we obtain the following:

Corollary 11. The mapping class groups $\Gamma_{h}$ are not weakly boundedly generated for any $h \geq 1$.

Such a statement, with weak bounded generation replaced by bounded generation, was first proved by Farb-Lubotzky-Minsky [8 using the theory of $p$-adic analytic groups.

One can contrast the above results on the existence of homogeneous quasihomomorphisms on mapping class groups with the results of Burger and Monod [6], who proved that lattices in higher rank Lie groups do not admit any homogeneous quasi-homomorphisms; compare also [19]. Because of their results, the special case of Theorem 7 proved in [7] suffices to prove the non-arithmeticity of mapping class groups in a way which is different from Ivanov's proofs, except for the fact that one has to appeal to one of the usual arguments to exclude rank 1 lattices, see the discussion in Chapter 9 of [12. As explained by Bestvina and Fujiwara [3], using the existence of quasi-homomorphisms on all non-virtually-Abelian subgroups of mapping class groups in conjunction with the results of Burger and Monod gives a new proof of the superrigidity result of Farb and Masur [9], which is independent of [9] and [13. This argument does not require the full strength of Theorem 10] as one only needs the non-triviality (rather than the infinite-dimensionality) of the space of homogeneous quasi-homomorphisms on every non-virtually-Abelian subgroup of a mapping class group. In fact, the above proof of Corollary 11 is the

\footnotetext{
${ }^{6}$ which we identify with its mapping class
} 
only application I know of the infinite-dimensionality of the space of homogeneous quasi-homomorphisms on $\Gamma_{h}$.

Remark 12. The discussion in this section applies to mapping class groups of surfaces with marked points and/or boundaries, but it does not apply to extended mapping class groups consisting of isotopy classes of not necessarily orientationpreserving diffeomorphisms. In an extended mapping class group a Dehn twist is conjugate to its inverse, because it is a product of two involutions, see A'Campo [1]. Thus, every quasi-homomorphism defined on an extended mapping class group must vanish on a Dehn twist, cf. Example2.

\section{ACKNOWLEDGMENTS}

I am grateful to B. Farb for pointing out the question in [5] about the torsion lengths of elements of mapping class groups. Having answered this question in an ad hoc way, I was spurred to think systematically about such questions by the appearance of M. Korkmaz's preprint [15]. Thanks also to M. Burger for useful email communications.

\section{REFERENCES}

1. N. A'Campo, Monodromy of real isolated singularities, Topology 42 (2003), 1229-1240. MR 2004c:14011

2. C. Bavard, Longueur stable des commutateurs, Enseign. Math. 37 (1991), 109-150. MR 92g:20051

3. M. Bestvina and K. Fujiwara, Bounded cohomology of subgroups of mapping class groups, Geometry \& Topology 6 (2002), 69-89. MR 2003f:57003

4. V. Braungardt and D. Kotschick, Clustering of critical points in Lefschetz fibrations and the symplectic Szpiro inequality, Trans. Amer. Math. Soc. 355 (2003), 3217-3226. MR 2004b:57034

5. T. E. Brendle and B. Farb, Every mapping class group is generated by 3 torsion elements and by 7 involutions, Preprint arXiv:math.GT/0307039 v2 24Jul2003.

6. M. Burger and N. Monod, Bounded cohomology of lattices in higher rank Lie groups, J. Eur. Math. Soc. 1 (1999), 199-235; Erratum ibid. 1 (1999), 338. MR 2000g:57058a

7. H. Endo and D. Kotschick, Bounded cohomology and non-uniform perfection of mapping class groups, Invent. Math. 144 (2001), 169-175. MR 2001m:57046

8. B. Farb, A. Lubotzky and Y. N. Minsky, Rank one phenomena in mapping class groups, Duke Math. J. 106 (2001), 581-597. MR 2001k:20076

9. B. Farb and H. Masur, Superrigidity and mapping class groups, Topology 37 (1998), 11691176. MR 99f:57017

10. R. E. Gompf, A topological characterization of symplectic manifolds, arXiv:math.SG/0210103 v1 7 Oct2002.

11. W. J. Harvey, Branch loci in Teichmüller space, Trans. Amer. Math. Soc. 153 (1971), 387399. MR 45:7046

12. N. V. Ivanov, Mapping class groups, in Handbook of geometric topology, North-Holland, Amsterdam 2002, pp. 523-633. MR 2003h:57022

13. V. A. Kaimanovich and H. Masur, The Poisson boundary of the mapping class group, Invent. Math. 125 (1996), 221-264. MR 97m:32033

14. M. Korkmaz, Stable commutator length of a Dehn twist, Preprint arXiv:math.GT/0012162 v2 9Jul2003.

15. M. Korkmaz, On a question of Brendle and Farb, Preprint arXiv:math.GT/0307146 v2 11Jul2003.

16. D. Kotschick, On regularly fibered complex surfaces, Geometry \& Topology Monographs 2: Proceedings of the Kirbyfest (1999), 291-298. MR 2001f:14020

17. C. Maclachlan, Modulus space is simply connected, Proc. Amer. Math. Soc. 29 (1971), 85-86. MR 44:4202 
18. J. McCarthy and A. Papadopoulos, Involutions in surface mapping class groups, Enseign. Math. 33 (1987), 275-290. MR 89a:57010

19. N. Monod, Continuous bounded cohomology of locally compact groups, LNM 1758, Springer Verlag 2001. MR 2002h:46121

20. L. Mosher, Mapping class groups are automatic, Ann. of Math. 142 (1995), 303-384. MR 96e:57002

21. L. Polterovich and Z. Rudnick, Kick stability in groups and dynamical systems, Nonlinearity 14 (2001), 1331-1363. MR 2003d:37003

22. J. Powell, Two theorems on the mapping class group of a surface, Proc. Amer. Math. Soc. 68 (1978), 347-350. MR 58:13045

Mathematisches Institut, Ludwig-Maximilians-Universität München, Theresienstr. 39, 80333 München, Germany

E-mail address: dieter@member.ams.org 